\title{
INVESTIGACIONES
}

\section{SENTIDOS DE LA ENSEÑANZA MEDIA DESDE LA EXPERIENCIA ESCOLAR DE ESTUDIANTES DE LICEOS MUNICIPALES}

\author{
Meanings of secondary education from the school experience \\ of students of public schools
}

\author{
Walter Manuel Molina Chávez.
}

Universidad de Magallanes, Departamento de Ciencias Sociales, Casilla 113-D, Punta Arenas, Chile.

\section{Resumen}

En este artículo se aborda la compleja problemática de la construcción de sentidos de la enseñanza media en Chile. Específicamente, se presentan los resultados de una investigación cuyos propósitos centrales fueron: identificar, describir e interpretar las representaciones sociales sobre el sentido de la enseñanza media desde la experiencia escolar de los estudiantes de liceos municipales de distinto estrato socioeconómico (bajo, medio-bajo, medio, medio-alto) en la Región Metropolitana.

Los resultados de este estudio permitieron identificar cuatro tipos dominantes de representaciones sociales sobre el sentido de la enseñanza media: prepararse para el futuro, formarse como persona, tener más cultura y vivir la experiencia. Se trata de cuatro representaciones o modelos simbólicos que dan cuenta de un horizonte de sentido fragmentado en torno al cual se polarizan los diferentes modos en que viven la experiencia escolar los estudiantes que asisten a liceos municipales (públicos) de nuestro país. En este contexto, se concluye la necesidad de incorporar en el diseño de las actuales políticas educativas esta diversidad de representaciones sobre el sentido, lo cual puede contribuir, significativamente, a revitalizar el sentido personal y colectivo de este nivel educativo.

Palabras clave: sentido, representaciones sociales, enseñanza media municipal, experiencia escolar.

\begin{abstract}
This article tackles the difficult subjects of the construction of the sense of secondary education in Chile. Specifically, we present the results of a research, which main purposes were identify, describe and interpret social representations about the sense of high school education from the school experience of the students of public schools of different socioeconomic status (lower class, lower-middle class, middle class, upper-middle class) in the Metropolitan Region of Chile.

The results of this study, allowed us identify four prevailing types of social representations about the sense of secondary education: prepare for the future, build oneself as a person, be cultured, and live the experience. It is about four representations or symbolic models which show a horizon of fragmented sense in which are polarized the different ways the students of public schools live the school experience in our country. In this context, we conclude the need to incorporate to the design of the current educational politics this diversity of representations about the sense; which can contribute, significantly to revitalize the personal and collective sense of this educational level.
\end{abstract}

Key words: meanings, social representations, public high school education, school experience. 


\section{PRESENTACION}

En los últimos treinta y cuatro años la sociedad chilena ha sido objeto de un conjunto de transformaciones estructurales que han modificado radicalmente el campo económico, político, social, cultural y educativo. Estas transformaciones, analizadas en su conjunto, han impactado fuertemente en la configuración del actual sistema educativo y de sus principales problemas: bajos niveles de calidad, inequidad en el acceso, segmentación social de la oferta educativa, institucionalidad inadecuada para los actuales desafíos educativos, entre otros aspectos relevantes a considerar.

En este contexto de transformaciones globales de la sociedad, en especial de los radicales cambios en los fundamentos y fisonomía del sistema educativo chileno, la enseñanza media (y especialmente la enseñanza municipal) se encuentra en el centro del debate educativo; no sólo por encontrarse afectada por una grave crisis de sentido existencial de los actores educativos sino también se manifiesta en una crisis de funcionalidad del sistema en su conjunto. Lo anterior ha impactado directamente en el proceso de construcción de sentido subjetivo, es decir, las atribuciones de significado que los jóvenes estudiantes secundarios hacen sobre el sentido de la enseñanza media desde su propia experiencia escolar en liceos municipales.

Para diversos autores (Tenti 2000, 2002; Braslavsky 2001; Berger Filho 2002; Kessler 2002; Baeza 2005b, Ferreira 2006) esta encrucijada en la que se encuentra la Enseñanza Media en América Latina y particularmente en Chile se relaciona con tres fenómenos claves y fuertemente conectados: el proceso de masificación y obligatoriedad de la enseñanza media, la creciente tendencia a la segmentación social de la oferta educativa del nivel medio y, finalmente, la evidente falta de protagonismo de los jóvenes y estudiantes secundarios en los procesos de enseñanza-aprendizaje que se implementan en los liceos de nuestro país.

En este contexto analítico, Ferreira (2006: 2) sostiene que la escuela media aparece, paradójicamente, encomendada a una doble función social: favorecer, con la ampliación de su oferta, la incorporación de todos -y aun de los menos habilitados desde un punto de vista económico y social- y a su vez continuar con su mandato fundacional de seleccionar académica y culturalmente a los más aptos. De lo anterior se derivan algunos de los problemas centrales de los debates educativos actuales respecto a la enseñanza secundaria. Como, por ejemplo, la necesidad de redefinir su actual función y estructura, actualizar su misión social y potenciar su contribución específica a los propósitos sistémicos. Pero desde nuestra perspectiva de análisis, lo más relevante de estos debates educativos lo constituye la necesidad de reconocer y describir el sentido subjetivo que adquiere este nivel de enseñanza desde la experiencia escolar de los jóvenes que habitan cotidianamente el espacio escolar de la enseñanza media municipal en Chile.

En respuesta a los problemas más relevantes del nivel de enseñanza media, las políticas educativas públicas han dirigido su acción a promover un conjunto de transformaciones e innovaciones: cambios curriculares, mejoramiento de infraestructura educativa, expansión de su cobertura y además la implementación de la Jornada Escolar Completa (JEC).

No obstante lo anterior, y considerando que el objetivo originalmente planteado por el programa de mejoramiento educativo MECE-MEDIA fue superar la evidente crisis de sentido que afectaba a este nivel educativo desde los inicios de la década de los noventa, 
en la actualidad diversos autores e instituciones (Bellei 2000; Weinstein 2001; Cariola 2003; OCDE, 2004; Baeza 2005b; Consejo Asesor Presidencial 2006) sostienen que aún existen importantes problemas y desafíos por cumplir en materias tales como: bajos niveles de calidad en los procesos y resultados educativos, problemas de cobertura en los sectores más pobres de la sociedad, deserción escolar, bajos resultados en las pruebas estandarizadas, entre otros aspectos a considerar. Por ello, es relevante preguntarse si efectivamente la crisis de sentido percibida y diagnosticada al inicio de la década del noventa por los diferentes actores del sistema educativo ha sido superada o, por el contrario, persiste en el tiempo y más aún, también es factible que dicha crisis se haya profundizado, especialmente en los liceos de dependencia municipal.

Como una forma de presentar sinópticamente los diversos aspectos de esta investigación este artículo se ha organizado en cinco apartados. En el primero de ellos se presenta el problema de investigación. En segundo lugar, se enuncian brevemente algunos de los referentes teóricos que sustenta la investigación. Posteriormente se exponen las principales decisiones y opciones metodológicas del investigador. El cuarto apartado está referido a la presentación y discusión de los principales resultados de la investigación. Finalmente, se presentan las conclusiones más relevantes del estudio.

\section{EL PROBLEMA DE INVESTIGACION}

En Chile, a comienzos de la década de los noventa, los diversos actores del sistema educativo construyeron un amplio consenso en torno a que la Enseñanza Media se encontraría en una grave crisis de sentido (Cariola y Cox 1990; Mena et al. 1991; Lemaitre 1992; Mineduc 1994; Haye \& Pacheco 1995). Para algunos autores, esta crisis tiene dos componentes básicos a saber: "una crisis de relevancia y de calidad de la enseñanza media" (Cariola et al. 1990: 19). Ambos aspectos se hacen evidentes en un contexto de expansión sistemática de la matrícula y de masificación de este nivel educativo.

Como consecuencia de esos primeros diagnósticos, y teniendo en cuenta la importancia estratégica que tiene este tipo de enseñanza para los procesos de desarrollo social y en la trayectoria de vida de los propios jóvenes (Cepal 1992; López y Tedesco 2002), se realizan nuevas investigaciones, las que aportarían diversos antecedentes empíricos sobre los principales problemas de la enseñanza media. Desde una perspectiva oficial, la "crisis" se formulaba en los siguientes términos:

La enseñanza media, además de presentar problemas de inequidad, ineficiencia y falta de calidad similares a la básica, padece además de una severa crisis de orientación y de relación con su medio externo; esto es, con el sistema de educación superior, con el sector productivo y el mercado del trabajo, y con el mundo contemporáneo de la información y el conocimiento (Comisión Nacional para la Modernización de la Educación 1994: 37).

Las conclusiones de este informe señalan la urgente necesidad de implementar una estrategia de mejoramiento integral de la educación, capaz de orientarse a adecuar la enseñanza media a las demandas emergentes en el contexto de los cambios que se estaban produciendo en las esferas económicas, políticas y culturales de la sociedad.

Como podemos observar, en los diferentes diagnósticos las dimensiones de la crisis ya no sólo abarcan aspectos vinculados al funcionamiento interno del sistema educativo 
(sus insumos y resultados de aprendizaje), sino también se pone en cuestión su capacidad socializadora, y lo que es más grave aún, se encontraría afectada su capacidad de integración y movilidad social de la población escolar chilena.

En este complejo escenario educativo y social, el Ministerio de Educación diseñó en 1994 el Programa de Mejoramiento de la Calidad y la Equidad de la Enseñanza Media (MECE-MEDIA) como una forma de abordar los diferentes aspectos de la crisis a los que hacen alusión los diferentes estudios. Con la implementación de este programa se buscó superar los problemas que afectaban tanto a los procesos educativos como a la estructura de la enseñanza media, así como su débil relación con las necesidades del entorno. En el diagnóstico que da origen al programa de mejoramiento antes descrito, se establece lo siguiente: "la educación media está hoy en una grave crisis de sentido, tanto para la sociedad en su conjunto, como para los jóvenes que acceden a ella. Su estructura y currículos son anacrónicos; sus resultados son de pobre calidad; las oportunidades que ofrecen son altamente inequitativas" (MINEDUC 1994: 14). Frente a esta "crisis de sentido", el programa MECE-MEDIA orientó gran parte de sus recursos y estrategias a producir y articular una propuesta de renovación global de la enseñanza media, fundada en criterios de calidad y equidad.

No obstante lo anterior, y luego de más de diez años de implementación de diversas estrategias orientadas al mejoramiento y renovación de la enseñanza media, y específicamente concluido el programa MECE-MEDIA en el año 2000 (MINEDUC 2001), diversos autores (Bellei 2000; Weinstein 2001; Baeza 2002; Cariola 2003; OCDE 2004) sostienen que pese a las significativas mejoras de este nivel de enseñanza aún persisten importantes problemas y desafíos que cumplir en materias tales como: cobertura y deserción escolar, calidad de los aprendizajes, bajos resultados en las pruebas estandarizadas, entre otros aspectos a considerar. Lo anterior nos lleva a preguntarnos si esa "crisis de sentido" percibida en su momento por los diferentes actores del sistema educativo ha sido superada o, por el contrario, si se mantiene plenamente vigente o incluso se ha profundizado, especialmente en los liceos de dependencia municipal.

Recientemente, y en el contexto de las movilizaciones de los estudiantes secundarios a principios del año 2006, se construyó un cierto consenso en torno a que el sistema educacional, en todos sus niveles, depende del significado que la sociedad y los actores le asignen a la educación (Comisión Asesora Presidencial 2006: 58). Por ello, la pregunta por el sentido de la educación [en sus distintas variantes: ¿Para qué educar?; ¿Para qué asistir a la escuela?] es relevante en el actual debate educativo, $y$, por consiguiente, las posibles respuestas a esta pregunta desde la experiencia escolar de los diversos actores deben incidir decisivamente en la estructuración de las políticas educativas del futuro inmediato. Por ello, en este complejo escenario educativo se sostiene que el sentido de la enseñanza municipal, construido desde la experiencia escolar de los estudiantes de liceos municipales, se expresa finalmente en representaciones sociales, las que a su vez constituyen un elemento mediador entre la experiencia escolar de los estudiantes y las políticas educativas implementadas en este nivel educativo, obstaculizando o facilitando con ello el logro de una enseñanza media con alta relevancia personal (y social), una alta calidad académica y una mayor equidad en la distribución social de sus resultados de aprendizaje.

Finalmente, el problema de investigación queda formulado en la siguiente pregunta de investigación: ¿Cuáles son las representaciones sociales sobre el sentido de la enseñanza media que construyen desde su experiencia escolar los estudiantes de liceos municipales en Chile? 


\section{PRINCIPALES REFERENTES TEORICOS DE LA INVESTIGACION}

Este trabajo de investigación se apoya en tres tradiciones teóricas relevantes para la investigación social y educativa. La primera tradición corresponde a la tradición filosófica, lingüística y sociológica que permitió acceder y delimitar la noción de sentido (Husserl 1991; Weber 1964; Flores 2001; Ríos 2004). El segundo referente teórico utilizado corresponde a la sociología de la experiencia escolar (Dubet 1991, 1994; Dubet \& Martuccelli 1996, 1998), la cual nos aporta una visión actualizada de la compleja relación entre el actor y el sistema escolar (y sus funciones) en el marco de la masificación y obligatoriedad de los procesos de escolarización secundaria en el mundo contemporáneo. En tercer lugar, la teoría de las representaciones sociales (Moscovici 1961, 1979; Jodelet 1988) permitió reconocer y abordar el proceso de configuración del sentido de la enseñanza media como el resultado de una construcción colectiva capaz de involucrar a todos los participantes del proceso. A continuación realizaremos una sintética revisión de los principales constructos teóricos en los que se apoya esta investigación.

\section{- $\quad$ NOCION DE SENTIDO}

La pregunta por el sentido es una interrogante siempre compleja que no tiene respuesta directa y mucho menos única. En un primer intento de respuesta emerge una red de conceptos de difícil delimitación, los cuales son utilizados de distinta forma en diversas disciplinas: finalidad, significado, propósitos, intencionalidad, intereses, motivos, entre otros. Dada esta dispersión conceptual, se sigue la indicación de Holzapfel (2005) para quien el sentido guarda relación principalmente con tres ámbitos: semántico, existencial y metafísico. En el ámbito semántico, el sentido es el significado de una palabra. Desde el ámbito existencial el sentido hace directa referencia a una acción o una decisión. Y específicamente, se refiere a la justificación y la orientación que el sujeto le atribuye a ambas. Finalmente, en el ámbito metafísico el sentido guarda relación con el trasfondo o la pregunta por la posibilidad de un sentido absoluto o incluso del sinsentido de las acciones humanas.

En este contexto de reflexión y considerando que el foco de nuestra investigación es el sentido de la enseñanza media que construyen los jóvenes que asisten a liceos municipales, se ha optado por comprender el sentido desde dos espacios principales: el ámbito semántico, es decir, el significado que atribuyen los sujetos educativos al sentido de la enseñanza media municipal; y, por otra, se explora el sentido desde el ámbito existencial, es decir, interesa el modo en que los estudiantes secundarios justifican y representan la orientación o finalidad de este nivel de enseñanza desde su experiencia escolar en liceos municipales.

\section{- NOCION DE EXPERIENCIA ESCOLAR}

La noción de experiencia tiene diversos desarrollos teóricos en las ciencias sociales. En filosofía, por ejemplo, se ha discutido largamente acerca del papel que le cabe a la experiencia en la construcción o adquisición del conocimiento. En otras disciplinas, en cambio (como la antropología, psicología o sociología), se ha abordado la experiencia en directa vinculación con las formas de adquisición y expresión de los patrones culturales, 
de las vivencias personales, de las motivaciones y, finalmente, como manifestación del proceso individual de interiorización de normas y valores sociales. En ambos casos la noción de experiencia emerge como una fuente primaria de la construcción del sentido cultural.

En este mismo contexto analítico, la experiencia escolar queda definida como: "la vertiente subjetiva del sistema escolar; más exactamente, es la manera cómo los actores sociales se representan y construyen este sistema, definen cada uno de los elementos, administran o gestionan -para ellos- sus articulaciones" (Dubet 1991: 24). En este pasaje de la reflexión queda claramente establecida la relación tensional que existe (en el seno del pensamiento sociológico) entre sistema y actor.

\section{- NOCION DE REPRESENTACIONES SOCIALES}

En el marco de la presente investigación se ha optado por utilizar la noción de representaciones sociales, por cuanto permite aproximarnos desde el conocimiento cotidiano que construyen los jóvenes estudiantes de liceos municipales sobre el sentido de la enseñanza media. Por "representación social" entendemos un tipo de conocimiento de sentido común que organiza conocimientos, informaciones, imágenes y actitudes sobre un objeto social determinado (en nuestro caso el sentido de la enseñanza media). De esta forma, si encaramos en forma pasiva, la noción de representación se capta como el reflejo en la conciencia individual o colectiva de un objeto, es decir, un haz de ideas exteriores a ella. Sin embargo, la analogía con una fotografía tomada y registrada en el cerebro resulta fascinante, pero incompleta e insuficiente para dar cuenta de la singularidad de una representación social.

\section{ENFOQUE, MATERIAL Y METODO DE INVESTIGACION}

En un contexto de diversidad paradigmática, hemos optado por un enfoque cualitativointerpretativo, por cuanto esta perspectiva de análisis supone adentrarse en la experiencia social de los sujetos; bajo una actitud fenomenológica, de respeto y apertura. Siempre atentos a escuchar los relatos de los sujetos entrevistados, donde lo central no será la(s) pregunta(s), sino las respuestas y las significaciones que en éstas subyacen, privilegiando los datos de la experiencia.

- Estrategia de muestreo utilizada en el estudio. La investigación utilizó una estrategia de mиеstreo estructural (Canales 1991) para seleccionar los liceos estudiados. Idéntico procedimiento se utilizó para seleccionar a los estudiantes entrevistados. En este contexto metodológico, la muestra del presente estudio se estructuró a partir de los siguientes criterios de elección definidos por el investigador:

* Los participantes de este estudio fueron 32 estudiantes de $4^{\circ}$ año de enseñanza media. Estos estudiantes pertenecen a liceos municipales localizados en cuatro comunas de la Región Metropolitana, Chile.

* Que los participantes estén distribuidos proporcionalmente entre hombres y mujeres. En cada liceo se seleccionó por orden de lista, y entre los asistentes el día de la entrevista, 
a cuatro hombres y cuatro mujeres. Se seleccionaron cuatro comunas y respectivamente cuatro liceos -representativos- de diferentes estratos socioeconómicos.

* Las características más relevantes de los establecimientos seleccionados son las siguientes:

\begin{tabular}{|c|c|c|c|c|c|c|c|}
\hline $\begin{array}{l}\text { Estableci- } \\
\text { miento }\end{array}$ & Comuna & Dependencia & $\begin{array}{c}\text { Grupo } \\
\text { Socio- } \\
\text { económico }\end{array}$ & $\begin{array}{c}\text { Número } \\
\text { alumnos } \\
4^{\circ} \text { Medio } \\
\text { C-H (2003) }\end{array}$ & $\begin{array}{l}\text { Número } \\
\text { Entrevista }\end{array}$ & $\begin{array}{l}\text { Puntaje } \\
\text { Simce } \\
\text { Lenguaje } \\
(2001)\end{array}$ & $\begin{array}{c}\text { Puntaje } \\
\text { Simce } \\
\text { Matemática } \\
(2001)\end{array}$ \\
\hline LICEO D & San Ramón & $\mathrm{MD} * *$ & $\begin{array}{c}\text { A } \\
\text { (Bajo) }\end{array}$ & 40 & 8 & 206 & 207 \\
\hline LICEO C & La Pintana & $\mathrm{MD} * *$ & $\begin{array}{c}\text { B } \\
\text { (Medio Bajo) }\end{array}$ & 38 & 8 & 231 & 211 \\
\hline LICEO B & La Florida & $\mathrm{MC}^{* *}$ & $\begin{array}{c}\mathrm{C} \\
\text { (Medio) }\end{array}$ & 98 & 8 & 252 & 241 \\
\hline LICEO A & Las Condes & $\mathrm{MCC}^{* *}$ & $\begin{array}{c}\text { D } \\
\text { (Medio alto) }\end{array}$ & 21 & 8 & 273 & 257 \\
\hline
\end{tabular}

* El grupo socioeconómico al que pertenece el establecimiento sigue la clasificación del Simce 2001: A (bajo), B (Medio-Bajo), C (Medio); D (Medio-Alto) y E (Alto). Cabe señalar que no existen establecimientos municipales que atiendan población escolar del estrato socioeconómico alto.

** Dependencia: Es el tipo de administración. MD: Municipal sin financiamiento compartido. MC: Corporación municipal sin financiamiento compartido. MCC: Corporación municipal con financiamiento compartido.

- Técnicas de producción y análisis de datos. La principal herramienta de producción de datos fue la entrevista en profundidad, por cuanto esta técnica de investigación social tiene la potencialidad de acceder a los significados que los actores sociales investigados construyen sobre el/los sentido(s) de la enseñanza media municipal.

Por otra parte, el material textual producido a través de las entrevistas fue analizado con herramientas provenientes de la semántica estructural (Greimas 1966). Como sabemos, este autor integra entre sus supuestos teóricos y metodológicos diversos aspectos de la linguiística estructural (Saussure 1945). Entre los conceptos que Greimas recoge y aplica en su modelo de análisis semántico, encontramos aspectos centrales como los referidos al signo lingüístico, a su naturaleza y a sus relaciones con otros signos.

De este modo, Greimas (1976) plantea que la lengua está hecha de oposiciones, por lo tanto de continuidades y discontinuidades. En este contexto, es posible comprender la concepción de estructura que define del siguiente modo: "es la presencia de dos términos y de la relación entre ellos existente" (Greimas 1976: 28). Esta distinción implica dos consecuencias para el análisis semántico: un solo término-objeto no conlleva significación y la significación presupone la existencia de la relación, es decir, la condición 
necesaria de la significación es la aparición de la relación entre dos términos. En el plano lingüístico, a propósito de la relación, es posible identificar, por tanto, dos términos que poseen algo en común, dos términos en que puedan identificarse diferencias y la identificación de la doble naturaleza de la relación entre dos términos: conjunción y disjunción (continuidad y diferencia).

Lo anterior permite a Greimas definir las estructuras elementales del sistema semántico como un tipo de relación entre dos términos objeto. El hecho de que los términos por sí mismos no conllevan significación, se sustenta en una concepción de lengua en la cual a ésta no se la considera como un sistema de signos, sino como una trabazón de estructuras de significación.

\section{RESULTADOS DE LA INVESTIGACION}

En esta sección se presentan los resultados más relevantes obtenidos en el proceso de análisis estructural del material reunido a partir de 32 entrevistas en profundidad realizadas a igual número de estudiantes de cuarto año de enseñanza media pertenecientes a cuatro liceos municipales de diferentes comunas de la Región Metropolitana.

Tal como se indicó en la sección de referencias teóricas de nuestra investigación, el contenido informacional de una representación social se relaciona básicamente con el modo en el que están organizados los conocimientos e informaciones que poseen individuos o grupos con respecto a un objeto social determinado, en nuestro caso, el sentido de la enseñanza media municipal. Por ello, este saber de sentido común, al estar constituido (y expresado) por medio de palabras, conceptos y términos, se presta para realizar un primer nivel del análisis semántico estructural, es decir, la identificación de las disyunciones u oposiciones de base sobre las cuales se articula globalmente el discurso de los sujetos sobre el objeto de la representación social.

En este contexto de análisis, cabe recordar que la semántica estructural es una técnica de análisis del discurso, o sea, de lo que los individuos dicen de la realidad y no de la realidad misma. Por ello, esta metodología de análisis es altamente apropiada para indagar sobre las representaciones que los individuos se hacen de una realidad particular y de los sistemas de sentido que orientan sus percepciones, sus pensamientos y que finalmente guían su acción social o propiamente escolar.

En términos globales, los resultados de esta investigación dan cuenta de la identificación de cuatro ejes semánticos que organizan el contenido de información y conocimientos de los sujetos respecto del sentido de la enseñanza media municipal. Estos ejes simbólicos emergentes son los siguientes: a) Eje semántico-temporal "Presente/ Futuro" b) Eje semántico "Sentidos globales de la enseñanza media" c) Eje semántico "Aportes de la enseñanza media" d) Eje semántico-espacial "Espacios de construcción de sentido". Considerando la necesidad de síntesis, en este artículo sólo presentaremos los resultados correspondientes a los dos ejes semánticos antes enunciados.

\subsection{Eje semántico temporal "Presentelfuturo" como articulador de la representación} social del sentido de la enseñanza media. Como se observa en los siguientes textos seleccionados, cuando a los entrevistados se les pregunta: ¿Cuál es el sentido de la 
enseñanza media para ti?, su repuesta se construye en torno a una clara disyunción temporal que opone presente y futuro de un modo radical:

El sentido de la enseñanza media es prepararte para el futuro, prepararte, prepararte pa' la universidad si queri estudiar en la Universidad y si no queris estudiar en la universidad dejarte con los conocimientos básicos que necesitai de educación, pa' trabajar y no estar perdio en la vida, eso es p' mi la enseñanza media. Y la enseñanza básica es como prepararte pa' la media, prepararte p' lo fuerte, p' lo que viene después (E5, LC, S, Hombre, p. 11).

Es que a uno le entrega mucho la enseñanza media, aparte de conocimientos, te entrega muchas otras cosas, te da como el empujoncito ahora para seguir la vida después, porque cuando uno está en básica es para que tú empieces, pero ahora es para que tú salgas adelante y empieces la vida, o sea se forme lo que va a ser tu vida después,.. (E2, LC, M, Mujer, p. 22).

En realidad el sentido para mí de la enseñanza media, es el sentido común que tienen todos los jóvenes, tengo que aprender, porque esta es la última parte, tengo que disfrutar porque si no voy a perder algo de mi vida, ya que estamos viviendo algo y hay que vivir el presente, uno no puede vivir un futuro ni un pasado. Yo estoy achacado porque tengo muchas cosas que hacer ahora, pero si pienso en el futuro me achaco más, por eso yo digo que hay que disfrutar la enseñanza media en este momento, las cosas que pueda ver, que pueda tocar, sentir de mi enseñanza, recibirlas con alegría y eso es lo que yo siento de la enseñanza media (E6, LP, L, Hombre, p. 14).

De este modo, el discurso de los estudiantes sobre el sentido de la enseñanza media emerge organizado en torno a un eje semántico temporal que se expresa en las siguientes estructuras semánticas paralelas:

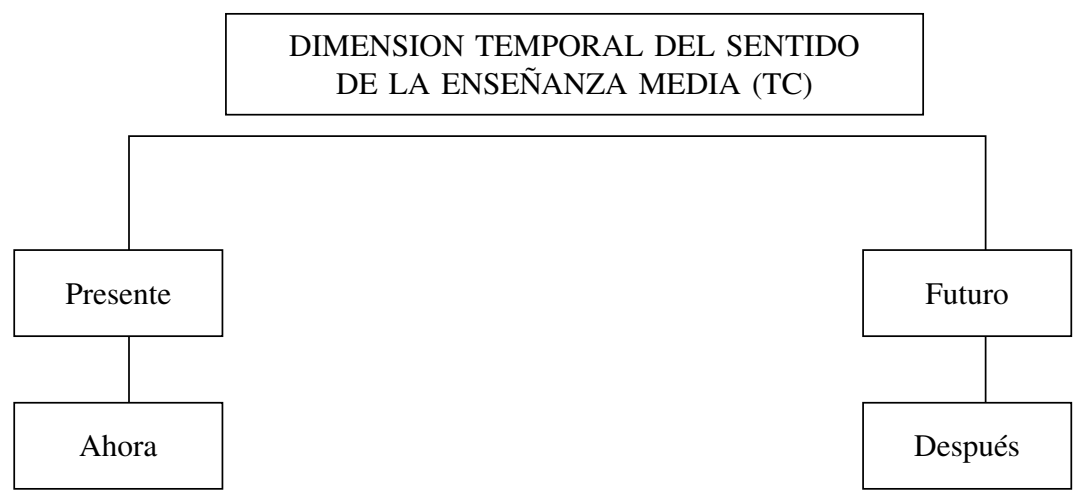

Como podemos observar, sobre este eje temporal las disyunciones: presente /futuro; ahora/después, sirven de elemento articulador y soporte desde el cual los estudiantes que asisten a los diferentes liceos municipales construyen los diversos sentidos de la enseñanza media.

Por ejemplo, cuando dicho discurso se apoya preferentemente en los términos presente o ahora, se destaca una dimensión temporal del sentido de la enseñanza media que se construye por el conjunto, experiencias, aportes y valoraciones actualizadas al 
momento presente, y que en consecuencia no necesariamente se proyectan hacia un tiempo futuro.

Desde esta perspectiva, la enseñanza media adquiere un sentido y un valor en el ahora, en el momento presente. Tal como lo indica un estudiante en su reflexión (muy personal) pero muy expresiva de esta tendencia: <por eso yo digo que hay que disfrutar la enseñanza media en este momento, las cosas que pueda ver, que pueda tocar, sentir de mi enseñanza, recibirlas con alegría y eso es lo que yo siento de la enseñanza media> (E6, LP, L, Hombre, p. 14). Lo anteriormente planteado nos muestra una valoración de la enseñanza media como una etapa actual de vida, que es por sí misma relevante y significativa en el contexto global de su ciclo de vida. Lo anterior, a pesar de ser valorado positivamente (y con matices diversos) por los estudiantes de todos los establecimientos, pero a la hora de responder a la pregunta por el sentido de la enseñanza media, los estudiantes se posicionan, claramente, en uno u otro polo de la disyunción temporal presente/futuro; produciendo con ello dos tendencias claramente diferenciadas: una tendencia presentista (el sentido emerge y se valida en el aquí/ahora) que indica que la enseñanza media tiene un/os sentido(s) que no se construyen o no se configuran polarizados hacia un momento futuro (y hacia unas finalidades "últimas").

Por otra parte, cuando los estudiantes enfatizan los términos futuro o después se hace alusión una dimensión del sentido de la enseñanza media que se proyecta hacia un tiempo desconocido e incierto, el cual es muy valorado y central en el discurso del segmento de estudiantes pertenecientes a liceos municipales considerados de estrato socioeconómico medio y medio-alto (La Florida y Las Condes respectivamente). Estas dos tendencias las podemos observar en el siguiente esquema:

\section{Esquema 1}

Eje semántico temporal Presente/Futuro

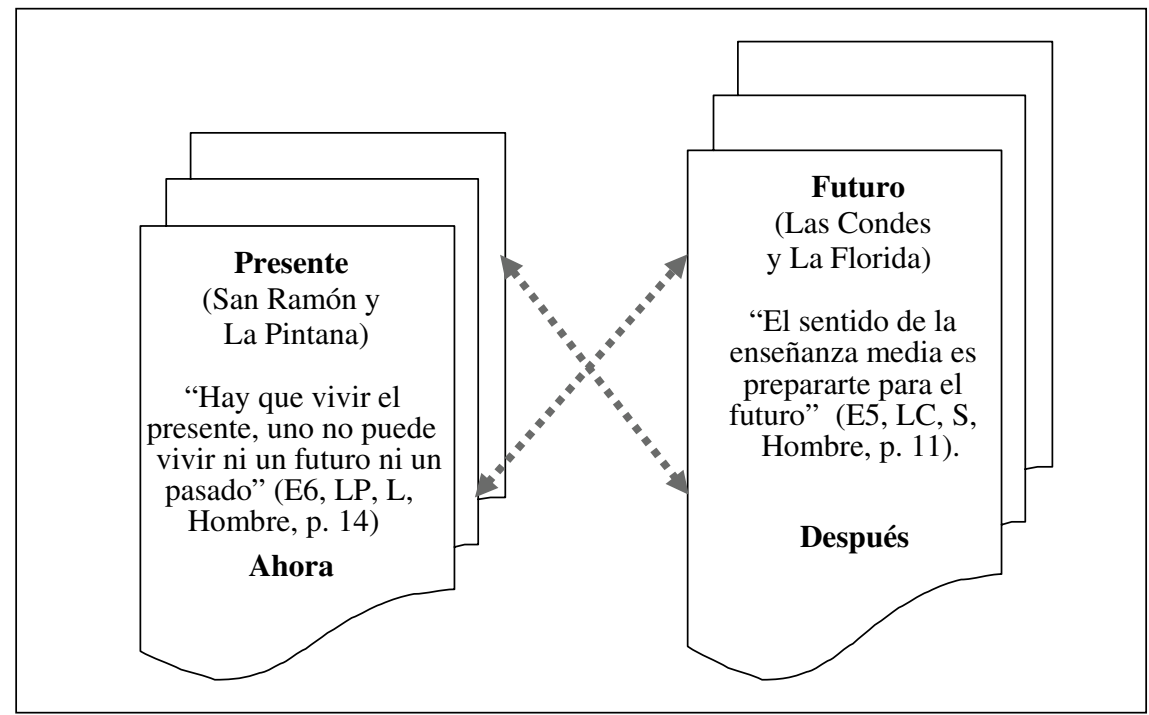


Como se ha descrito anteriormente, las estructuras semánticas que emergen en los textos analizados dan cuenta de una radical oposición entre presente y futuro. Esta disyunción madre es un espacio simbólico que se constituye en el soporte temporal desde el cual se construyen los diferentes sentidos sobre la enseñanza media. Esta disyunción temporal, a nuestro juicio, es una potente clave analítica que permite comprender el proceso de construcción de sentido que llevan a cabo los estudiantes en el contexto de una sociedad en mutación cultural.

Al respecto, diversos autores (Elias 1990; Lechner 2003) han señalado que la dimensión temporal ocupa un lugar central en los actuales procesos de cambio cultural. Estos cambios estarían marcados por la retracción o adelgazamiento del tejido institucional de la sociedad. A la base de estos procesos de retracción social y política habría un repliegue temporal que ha sido descrito del siguiente modo:

Vivimos en el presente como tiempo único. La aceleración vertiginosa del ritmo de vida diario es impulsada por tendencias de época: simultaneidad creada por la globalización, la mediatización de la comunicación social, la velocidad de las imágenes y la realidad "en vivo", la flexibilización del trabajo y la satisfacción inmediata del consumo [real o simbólico]. Estos cambios tienden a vaciar al principal andamiaje del tiempo largo: las instituciones. El tiempo lento de la institucionalidad parece obsoleto de cara al ritmo que la televisión y las encuestas de opinión imprimen al debate público. Ellas dilatan la realidad y aceleran la urgencia de los problemas, generando la impaciencia del público. Frente al tiempo del mercado -la contingencia-, el tiempo de la política sería el de la perspectiva... en realidad, la política -entre otras instituciones- se juega en el manejo del tiempo (Lechner 2003: 19).

Desde nuestra perspectiva, esta reflexión sobre el tiempo y de la necesidad de su dominio en el campo político es perfectamente aplicable al campo educativo, sobre todo si consideramos lo planteado por Laïdi (2000), quien sostiene: Sólo el dominio del tiempo permite a los hombres dejar de creer que son juguetes del azar. Sólo el dominio del tiempo les permite acceder a una forma de existencia donde ellos deciden colectivamente su destino (Laïdi 2000: 94).

De acuerdo al autor citado, sería tarea de la política contrarrestar la urgencia de la realidad inmediata mediante un tiempo histórico, es decir, con perspectiva de largo plazo. De este modo, la historicidad entrelaza discontinuidades y duración, experiencias aprendidas con horizontes de futuro. Estos horizontes no son proyecciones de metas ni planes a cumplir, más bien son "constructos" o apuestas acerca del sentido que atribuimos al trayecto realizado [o a una parte de él] y a las promesas de un mañana mejor. Visto así, hacer política [educativa] consiste en producir los horizontes de sentido que permiten a los actores sociales poner las cosas en perspectiva. A nuestro juicio, lo que ha sido planteado por varios autores (Laïdi 2000; Lechner 2003) para el campo de la política es perfectamente aplicable al campo educativo y específicamente al ámbito del diseño de políticas públicas en educación.

4.2. Eje semántico: Sentidos globales de la enseñanza media. Sobre el plano temporal antes descrito emerge un segundo eje semántico que hemos denominado directamente "los sentidos de la enseñanza media". Este eje simbólico condensa diferentes niveles de análisis de la representación social sobre el sentido que construyen los estudiantes entrevistados y que pertenecen a los diferentes liceos municipales estudiados. 
A continuación se presentan las estructuras semánticas paralelas y jerarquizadas que dan cuenta de los sentidos globales construidos por los estudiantes en torno al sentido de la enseñanza media municipal:

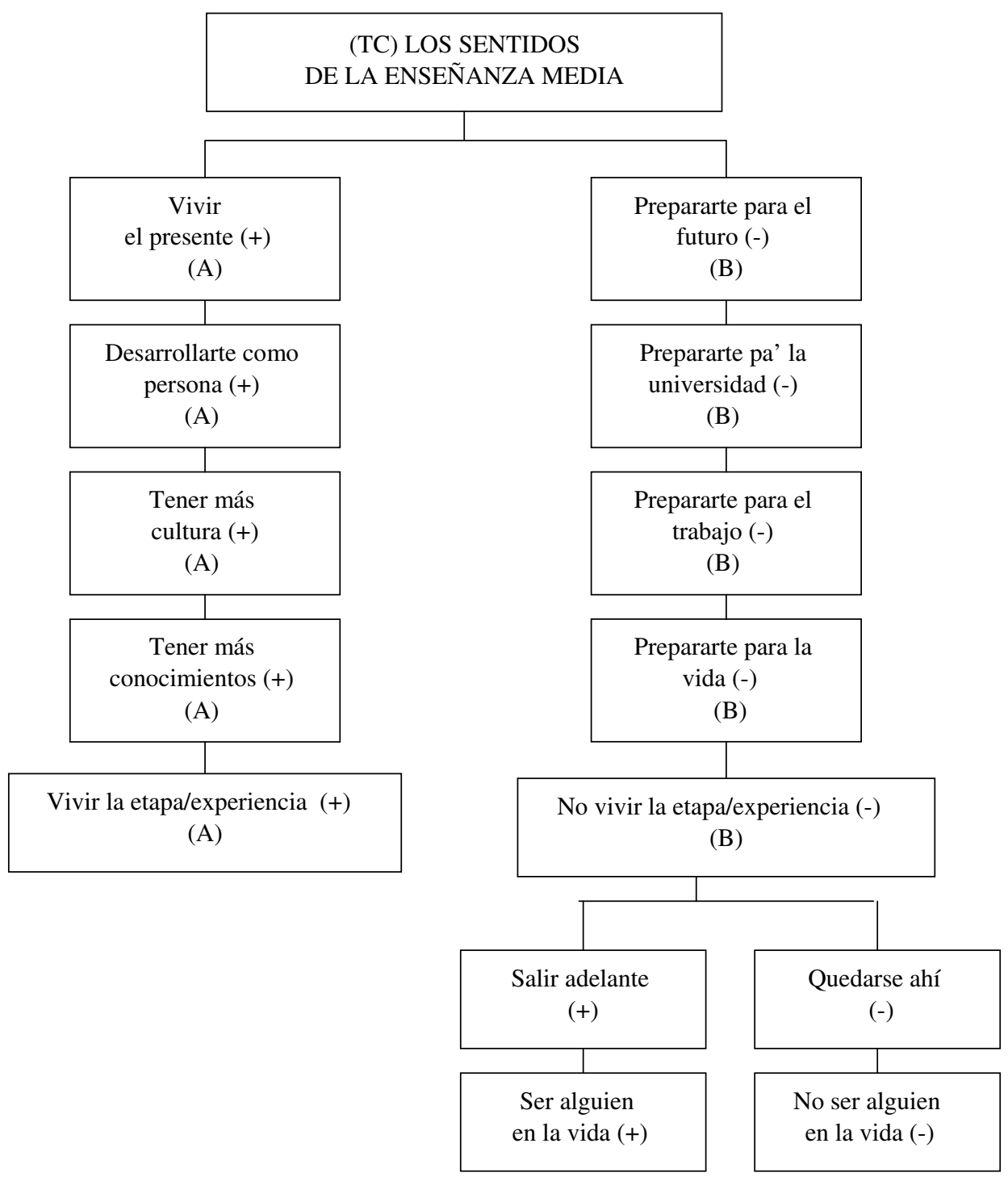

Como se puede observar, en el caso de los términos "A" de la disyunción, el horizonte de sentido construido por los estudiantes está circunscrito a un tiempo presente desde donde emerge una serie de términos objetos o realidades que enfatizan el sentido de la enseñanza media municipal como un conjunto de oportunidades actuales, evidentes y valoradas positivamente por los estudiantes: "vivir el presente", "tener más cultura", "obtener información y que se condensan en la unidad de significado", "vivir la experiencia" 
(estudiantil). Por otra parte, en el polo "B" de las disyunciones hemos agrupado aquellos términos o lexemas que enfatizan el sentido de la enseñanza media como un conjunto de posibilidades y desafíos concretos para el futuro, pero cuyos resultados finales son inciertos: prepararte para el futuro, prepararte pa' la universidad, ayudarte a obtener un trabajo estable y genéricamente prepararte para la vida.

Desde un punto de vista valorativo, los términos "A" tienden a consensuar una valoración positiva de la enseñanza media como una etapa importante en la vida de los estudiantes entrevistados y junto con ello se destacan los múltiples aportes más vivenciales y simbólicos que la enseñanza media realiza en el presente (o tal vez en el corto plazo) a la formación de los estudiantes que asisten y egresan de este nivel educativo.

En el otro extremo "B" de la oposición están los términos que expresan los sentidos de la enseñanza media en un escenario futuro, los cuales pese a ser enunciados como desafíos personales altamente deseados. Sin embargo, estos términos tienen asociado un sesgo de duda e incertidumbre en relación a sus posibilidades reales de consecución. De este modo, en diversos pasajes de los textos analizados, son enunciados múltiples aspectos débiles de la enseñanza recibida, la mayoría de los cuales son percibidos como obstáculos presentes en el entorno sociocultural que dificultan -alcanzar lo que quiero-, es decir, la realización personal de los estudiantes de la enseñanza media municipal queda en entredicho.

A continuación presentamos una estructura semántica cruzada que pone en interacción dos realidades madres que sintetizan el proceso de construcción de sentido de los estudiantes de cuarto año medio que asisten a liceos municipales de distinto estrato socioeconómico.

4.3. Estructura semántica cruzada sobre el eje semántico "Los sentidos de la enseñanza media”. La estructura semántica propuesta se constituye de un eje semántico temporal (vertical) y un eje horizontal que se opone: etapa / fin, es decir, cuando los estudiantes se representan o elaboran el sentido de la enseñanza media, lo consideran ya sea como una etapa por la cual se debe transitar y por otra parte el sentido se construye en torno a las finalidades de la experiencia escolar y de la propia escuela, las que se realizan en un escenario futuro de largo plazo. La estructura cruzada resultante de nuestro análisis es la siguiente: 


\section{(TC) SENTIDOS DE LA ENSEÑANZA MEDIA}

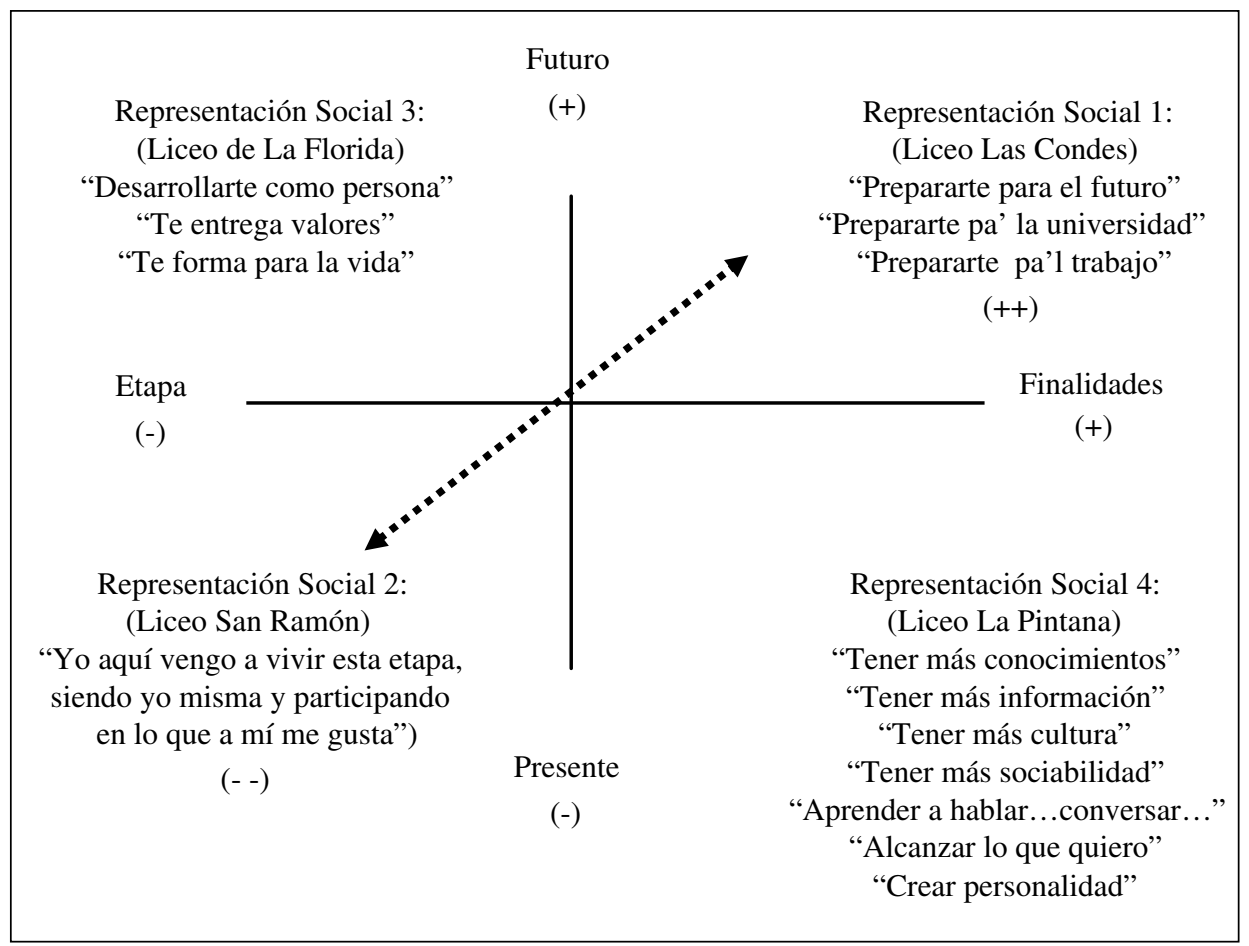

En el cuadro anterior se muestra la intersección de dos tipos de realidades madres que emergen en el discurso de los estudiantes de liceos municipales: el eje temporal, expresado en la tensión presente-futuro y el eje semántico -los sentidos de la enseñanza media- y que se expresa en la disyunción Vivir la etapa $\mathrm{v} / \mathrm{s}$ Alcanzar lo que quiero (las finalidades).

Como podemos observar, al cruzar estos dos ejes se configuran cuatro realidades o representaciones sociales sobre el sentido de la enseñanza media. En la Representación 1, el sentido emerge con una orientación clara al futuro y es explicitada en demandas específicas que le hacen los jóvenes a la enseñanza media (Prepararte pa' la universidad, prepararte pa'l trabajo, etc.). En el polo opuesto, surge la Representación 2, la cual revela un sentido construido en torno al presente y como necesidad existencial de vivenciar la experiencia escolar secundaria. Desde esta configuración de sentido, el aporte principal que la enseñanza media les hace a los jóvenes es en términos de sociabilidad, de reconocimiento y de canalización de sus necesidades de construcción de identidad juvenil.

En este punto existe una gran coincidencia con los resultados de otros estudios (Salinas \& Franssen 1997: 42) que evidencian que esta tendencia manifiesta la incapacidad del liceo o la escuela de constituirse en un espacio propiamente educativo, que acoja la sociabilidad juvenil sin dejarse desbordar por ella. Por su parte, la Representación 3 se construye de cara al futuro; pero lo que fundamentalmente dota de sentido a su experiencia escolar 
media es la promesa y realidad de desarrollarte como persona, lo cual incluye demandas y expectativas más de orden valórico y formativo. Adquirir conocimientos instrumentales (útiles para el futuro) sería algo que llegaría en segundo plano. En consecuencia, los logros académicos, en el liceo municipal, son representados por los sujetos como resultado directo del esfuerzo personal que cada uno de los estudiantes le pone a la tarea de aprender.

Finalmente, la Representación social 4 expresa un modelo simbólico sobre el sentido que se forma en torno a una de las dimensiones claves de la experiencia escolar y de las funciones de la escuela, como es la demanda de formación del sujeto, donde lo relevante para los estudiantes es tener más información, tener más cultura, tener más sociabilidad, entre otros sentidos más concretos (aprender a hablar, aprender a conversar, desenvolverse ante los demás, etc.). Todo esto permite a cada alumno alcanzar lo que cada uno verdaderamente quiere, es decir, realizarse como persona y fundamentalmente "salir adelante". Las cuatro tendencias anteriormente descritas permiten sostener la existencia de cuatro tipos de modelos simbólicos sobre el sentido de la enseñanza media municipal y que se sintetizan en el siguiente cuadro:

TIPOS DE REPRESENTACION SOBRE EL SENTIDO DE LA ENSEÑANZA MEDIA REPRESENTACION SOCIAL 1: El sentido de la enseñanza media es "prepararte para el futuro" REPRESENTACION SOCIAL 2: El sentido de la enseñanza media es "formarte como persona" REPRESENTACION SOCIAL 3: El sentido de la enseñanza media es "tener más cultura" REPRESENTACION SOCIAL 4: El sentido de la enseñanza media es "vivir la experiencia"

Cabe señalar que pese a que cada una de estas representaciones sobre el sentido se desarrolla al interior de un tipo de liceo analizado, lo cual no significa que se correspondan absolutamente con los discursos de todos los estudiantes de ese liceo en particular, por ello, más bien nos parece razonable sostener que los 32 estudiantes entrevistados se agrupan en cuatro "tipos ideales" de representación sobre el sentido, los cuales pueden coexistir (y de hecho coexisten) al interior de un mismo liceo e incluso al interior de una misma sala de clases.

En consecuencia, dichos sentidos generales más que correlacionarse directamente y linealmente con un tipo de liceo municipal perteneciente a un estrato socioeconómico específico (Bajo, medio-bajo, medio y medio-alto) dan origen a tendencias o figuras de sentido en torno a las cuales se polariza el discurso (y la acción) de los estudiantes y que como podemos observar coexisten al interior de un mismo liceo municipal, de una misma sala de clases.

Como se pudo observar, las representaciones sociales sobre el sentido de la enseñanza media identificadas en el cuadro anterior están organizadas a partir de diversas estructuras semánticas, paralelas y cruzadas, las cuales cumplen la función de dar forma al rico contenido informacional manifestado en el discurso de los estudiantes secundarios.

\section{CONCLUSIONES}

Una de las conclusiones más significativas de nuestra investigación consiste en haber identificado y descrito cuatro representaciones sociales sobre el sentido de la enseñanza media desde la perspectiva de la experiencia escolar de los estudiantes de 
liceos municipales: (1) "prepararte para el futuro" (2) "desarrollarte como persona" (3) "tener más cultura" y (4) "vivir la experiencia/etapa". En este contexto, se sostiene que el sentido que los jóvenes construyen en torno a la enseñanza media se manifiesta en representaciones sociales que pueden estar mediando su experiencia escolar cotidiana al interior de los establecimientos a los que asisten, obstaculizando o facilitando con ello el logro de una enseñanza media con alta relevancia social (y personal), alta calidad académica y con resultados de aprendizaje que contribuyan significativamente a una inserción de los jóvenes en los diferentes planos de la vida social.

Por otra parte, esta diversidad de representaciones sociales identificadas en nuestro estudio se corresponde con lo que Kessler (2002) denomina una experiencia escolar fragmentada en la escuela secundaria argentina, es decir, la existencia al interior de un mismo nivel educativo de diversos modos de vivir y experimentar los procesos de escolarización obligatoria. Por ello, y en el marco de este proceso de fragmentación de la experiencia escolar, es posible sostener que el proceso de construcción de sentido de la enseñanza media municipal en Chile se encuentra igualmente fragmentado. Más concretamente, y en los términos planteados por Tenti (2000) para el caso de la escuela secundaria argentina, el sentido como obligación social (y jurídica) de asistir a la enseñanza media, o el sentido como la posibilidad estratégica de acceder a mejores condiciones de vida y el sentido como amor al conocimiento y al aprendizaje (Tenti 2000: 10). Como señala este mismo autor, estas tres disposiciones ideales típicas descritas por la literatura no están aleatoriamente distribuidas en la población, sino que por el contrario son el producto de un conjunto de factores interrelacionados. Entre ellos, cabe mencionar los factores culturales, las trayectorias individuales y el lugar que ocupan las personas en la estructura social.

Finalmente, se sostiene que al reconocer la pluralidad de sentidos que portan los estudiantes que asisten a la enseñanza media en Chile, es posible que las políticas educativas dirigidas a este nivel educativo promuevan acciones (y programas) que permitan integrar dichos sentidos en el rediseño y renovación de la enseñanza media chilena.

En este contexto de análisis surge la siguiente paradoja: si la enseñanza media tiene como principal sentido el "prepararse para el futuro", sus actuales niveles de logro académico amenazan seriamente el cumplimiento de dicho sentido primario destacado por los jóvenes estudiantes de liceos municipales. En la perspectiva de superar esta paradoja, el liceo municipal tendría entre sus principales desafíos la tarea de (re)construir el carisma y la fuerza movilizadora que tuvo la enseñanza pública en momentos claves del desarrollo de la sociedad chilena.

\section{BIBLIOGRAFIA}

Baeza, J. (2005a). Estudiantes de enseñanza media: ni mejores ni peores, sólo diferentes. En: Calidad en la Educación, Una publicación del Consejo Superior de Educación (CSE), $\mathrm{N}^{\circ} 23$, diciembre 2005.

Baeza, J. (2005b). Ser alumn@ de Educación Secundaria en América Latina: Conocimiento sociológico presente en internet. Investigación Documental sobre el conocimiento sociológico acumulado, 2004-2004, Santiago: UNESCO.

Bellei, C. (2003). Veinte años de políticas en educación media en Chile: 1980-2000. De la autorregulación del mercado al compromiso público. Santiago: UNESCO. 
Berger Filho, R. (2002). Buscando un nuevo sentido para la educación escolarizada. En: ¿Qué educación secundaria para el siglo XXI? Santiago: UNESCO/OREALC.

Braslavsky, C. (Org.) (2001). La educación secundaria, ¿cambio o inmutabilidad? Análisis y debate de procesos europeos y latinoamericanos contemporáneos. Argentina: IIPE/UNESCO/ Santillana.

Canales, M. (1994). El estudio de la realidad social con metodologías cualitativas. Santiago: Ministerio Secretaría General de Gobierno/ División de Organizaciones Sociales.

Cariola, L. y C. Cox. La educación de los jóvenes: crisis de relevancia y Calidad de la Educación media. En: Generación Compiladores (1990). Los jóvenes en Chile Hoy. Santiago: CIDE/ CIEPLAN/PSI PIRQUE/SUR.

Cariola, L., C. Bellei, I. Núñez (2003). Veinte años de Políticas en Educación Media. Santiago: UNESCO.

Cox, C. (2003). Políticas Educacionales en el cambio de siglo. La reforma del sistema escolar en Chile, Santiago: Editorial Universitaria.

Dubet, F., D. Martuccelli (1998). En la escuela. Sociología de la experiencia escolar. Buenos Aires: Losada.

Ferreira, H. (2006). Transformación de la educación media en la Argentina. Tensiones y conflictos en el diseño e implementación en la provincia de Córdoba. Argentina: Editorial Universidad Católica de Córdoba.

Greimas, A.J. (1971). Semántica estructural. Investigación metodológica. Madrid: Gredos.

Holzapfel, C. (2005). A la Búsqueda del sentido. Santiago: Editorial Sudamericana.

Husserl, E. (1991). La crisis de las Ciencias Europeas. Y la fenomenología transcendental. Barcelona: Editorial Crítica.

Kessler, G. (2002). La experiencia escolar fragmentada. Estudiantes y docentes en la escuela media de Buenos Aires. Argentina: IIPE-UNESCO.

Laïdi, Z. (1997). Un mundo sin sentido. México: Fondo de Cultura Económica.

Lemaitre, M.J. (1992). Los jóvenes y la enseñanza media. En: Rittershaussen y Scharager (editores). Análisis y proyecciones en torno a la educación media y el trabajo. Santiago: Corporación de Promoción Universitaria.

López, N., J.C. Tedesco (2002). Desafíos a la educación secundaria en América Latina. Revista de la Cepal No 76/2002: 55-69.

Martinic, S. (1992). Análisis estructural: presentación de un método para el estudio de lógicas culturales. Santiago: Centro de Investigación Desarrollo de la Educación.

Mena, Isidora y otros (Comp.). La juventud y la enseñanza media. Una crisis por resolver. Santiago: Corporación de Promoción Universitaria.

MINEDUC (1994). Fundamentos, estrategias y componentes del programa de Mejoramiento de la Calidad y Equidad de la Educación Media. Santiago de Chile: Ministerio de Educación.

MINEDUC (2001). Informe Preliminar de Cierre del Programa MECE Media. Santiago de Chile: Ministerio de Educación.

Moscovici, S. (1979). El psicoanálisis, su imagen y su público. Buenos Aires: Huemul.

OCDE (2004). Revisión de políticas nacionales de educación. Chile. París: Organización para la Cooperación y el Desarrollo Económico.

Piret, A., J. Nizet, E. Bourgeois (1996). L'Analyse Structurale. Une méthode d'analyse de contenu pour les sciences humaines. Bruxelles: De Boeck Université.

Ríos, T. (2004). La configuración de sentidos en un grupo de jóvenes liceanos de sectores populares: un acercamiento hermenéutico al mundo juvenil escolar. Tesis Doctoral. Santiago: Pontificia Universidad Católica de Chile.

Tenti, E. (2000). Culturas juveniles y cultura escolar. Buenos Aires: UNESCO. 
Weber, M. (1964). Economía y sociedad. Esbozo de sociología comprensiva. México: Fondo de Cultura Económica.

Weinstein, J. (2002). Educación e integración social: una visión desde la práctica de las políticas educativas. En: Hoy es mi tiempo. Una ventana a la esperanza. Santiago: Fondo de Solidaridad e Inversión Social. 